\title{
A aplicabilidade de terapia por vestes elásticas em crianças com paralisia cerebral
}

\author{
The applicability of elastic garment therapy in children with cerebral palsy \\ La aplicabilidad de la terapia con prendas elásticas en niños con parálisis cerebral
}

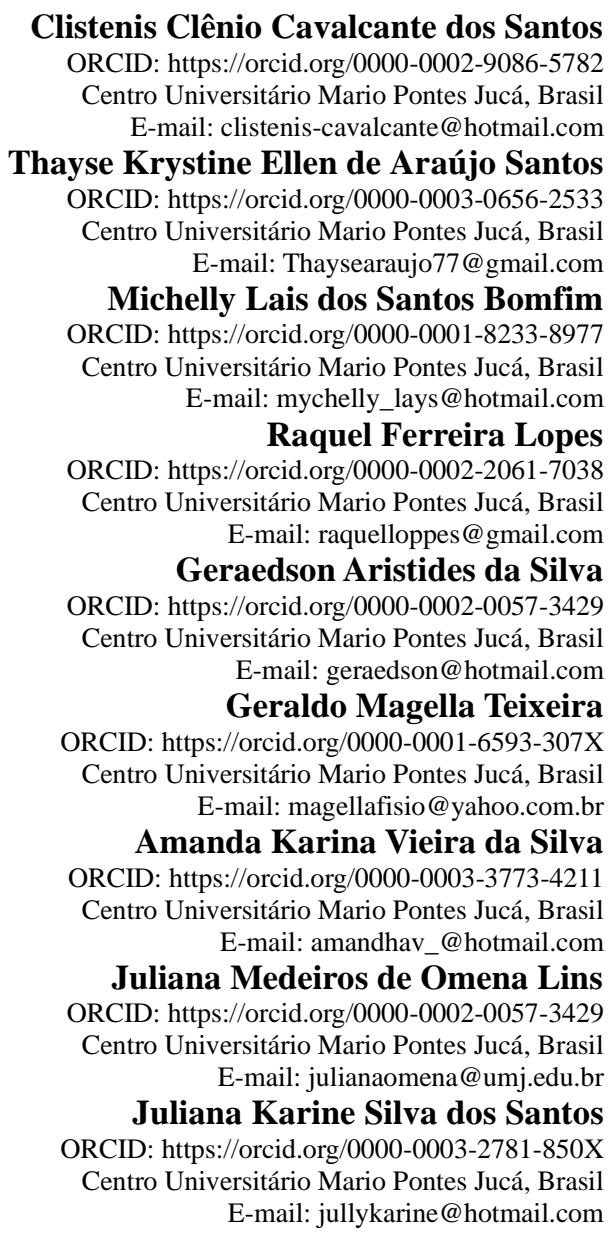

\begin{abstract}
Resumo
Introdução: A Paralisia Cerebral (PC) é determinada como encefalopatia crônica não progressiva que ocorre devido a uma lesão no encéfalo em desenvolvimento, comprometendo as funções motoras e a postura. No decorrer do processo de reabilitação dos pacientes com PC, podemos realizar a terapia por vestes elásticas, através de diversos protocolos, dentre eles o Pediasuit e Therasuit, com o principal objetivo terapêutico de promover o treinamento e desenvolvimento das funções neuromotoras. Objetivo: descrever os efeitos terapêuticos produzidos pelos métodos Pediasuit e Therasuit, em crianças com PC. Metodologia: Trata-se de uma revisão sistemática, realizada nas bases de dados eletrônicas SciELO, LILACS, PubMed e Periódicos CAPES, nos idiomas inglês, português e espanhol. Como estratégia de busca, utilizou as palavras chaves paralisia cerebral, vestes elásticas, pediasuit, therasuit, terapia neuromotora intensiva, de forma combinada ou isolada. Resultado: Ao identificar cerca de 21.116 artigos, estes foram submetidos aos critérios de inclusão e exclusão, restando cerca de 28 artigos para a composição deste artigo. Conclusão: Embora existam poucos artigos que abordem diretamente os métodos Pediasuit e Therasuit, em crianças com PC, identifica-se a existência de lacunas que necessitam serem respondidas nas próximas pesquisas, através de grupo controle, comparações das técnicas e a potencialidade da eficácia dos métodos citados, ainda que, nos faz identificar a existência de ganhos positivos no desenvolvimento infantil, destinados a avanços motores e posturais.
\end{abstract} Palavras-chave: Paralisia cerebral; Vestes elásticas; Pediasuit; Therasuit; Terapia neuromotora intensiva. 


\begin{abstract}
Introduction: Cerebral Palsy $(\mathrm{CP})$ is defined as a chronic non-progressive encephalopathy that occurs due to a lesion in the developing brain, compromising motor functions and posture. Through several protocols, including the Pediasuit and Therasuit, with the main therapeutic objective of promoting the training and development of neuromotor functions. Objective: to describe the therapeutic effects produced by the Pediasuit and Therasuit methods in children with CP. Methodology: This is a systematic review, carried out in the electronic databases SciELO, LILACS, PubMed and CAPES Periodicals, in English, Portuguese and Spanish. As a search strategy, the keywords cerebral palsy, elastic clothing, pediatric suit, therasuit, intensive neuromotor therapy, were used, in combination oral one. Result: Upon identifying about 21,116 articles, these were submitted to the inclusion and exclusion criteria, leaving about 28 articles for the composition of this article. Conclusion: Although there are few articles that directly address the Pediasuit and Therasuit methods in children with $\mathrm{CP}$, the existence of gaps that need to be answered in future research is identified, through a control group, comparisons of techniques and the potential effectiveness of the aforementioned methods, although it makes us identify the existence of positive gains in child development, aimed at advances motors and postures.
\end{abstract}

Keywords: Cerebral Palsy; elastics garments; Therasuit; Pediasuit; Intensive neuromotor Therapy.

\begin{abstract}
Resumen
Introducción: La Parálisis Cerebral (PC) se determina como una encefalopatía crónica no progresiva que se produce por una lesión en el cerebro en desarrollo, comprometiendo las funciones motoras y la postura. Durante el proceso de rehabilitación de los pacientes con PC, podemos realizar la terapia mediante prendas elásticas, a través de varios protocolos, entre ellos el Pediasuit y Therasuit, con el principal objetivo terapéutico de favorecer el entrenamiento y desarrollo de las funciones neuromotoras. Objetivo: describir los efectos terapéuticos producidos por los métodos Pediasuit y Therasuit en niños con PC. Metodología: Se trata de una revisión sistemática, realizada en las bases de datos electrónicas SciELO, LILACS, PubMed y Revistas CAPES, en inglés, portugués y español. Como estrategia de búsqueda se utilizaron las palabras clave parálisis cerebral, ropa elástica, traje pediátrico, traje terapéutico, terapia neuromotora intensiva, en combinación o sola. Resultado: Al identificar cerca de 21.116 artículos, estos fueron sometidos a los criterios de inclusión y exclusión, quedando cerca de 28 artículos para la redacción de este artículo. Conclusión: Aunque son pocos los artículos que abordan directamente los métodos Pediasuit y Therasuit en niños con PC, se identifica la existencia de brechas que necesitan ser abordadas en futuras investigaciones, mediante un grupo de control, comparaciones de técnicas y la potencial efectividad de los métodos. mencionado, sin embargo, nos hace identificar la existencia de ganancias positivas en el desarrollo infantil, orientadas a los avances motores y posturales.
\end{abstract}

Palabras clave: Parálisis cerebral; Prendas elásticas; Pediasuit; Therasuit; Terapia neuromotora intensiva.

\title{
1. Introdução
}

A Paralisia Cerebral (PC) é também nomeada como Encefalopatia Crônica não Progressiva (ECNP), representa um somatório de distúrbios do desenvolvimento e postura, ocasionado pela lesão cerebral não progressiva que acomete o Sistema Nervoso Central. A lesão, pode ocorrer nos períodos pré-natal, Peri natal ou pós-natal, sendo capaz de ser ocasionado por uma prematuridade, má formação fetal, parto instrumental, idade da genitora e pela duração do trabalho de parto (Mangilli, 2017; Santos et al., 2017).

As modificações provenientes da PC, ocasionam limitações e incapacidade, no público infantil em manter posturas e executar movimentos, devido as alterações corporais e estruturais, que são capazes de promover diversas manifestações clínicas (Santos et al., 2017). Os sinais clínicos da PC envolvem as alterações do tônus e presença de movimentos atípicos, incluindo o comprometimento motor baseado na distribuição topográfica (Cans et al., 2007).

A PC afeta as funções neuromusculares, esqueléticas e sensoriais ocasionando diferentes tipos de deficiências, dependo da distribuição topográfica acometida pela lesão (Gómez et al., 2016). Pode-se classificar a PC, pelos tipos de lesões resultantes de alterações no tônus muscular e desordem dos movimentos sendo elas: espástico, discinético ou atetóide, atáxico. Havendo também a classificação de leve, moderada ou grave (Queiroz et al., 2019).

Em casos de PC espástica, caracteriza-se pela presença de tônus elevado, aumento dos reflexos miotáticos, clônus, reflexo cutâneo plantar em extensão, sabendo que esta, se faz presente devido a existência de uma lesão no sistema piramidal (Scholtes et al., 2006). Já a discinética, identifica-se a existência de movimentos atípicos mais evidentes quando o paciente inicia um movimento voluntário produzindo movimentos e posturas atípicos, tônus instável, com a presença de movimentos 
involuntários e movimentação associada, em detrimento de uma lesão no sistema extrapiramidal, principalmente nos núcleos da base [corpo estriado - striatum e globo pálido, substância negra e núcleo subtalâmico] (Rosenbaum et al., 2007).

Nas crianças com quadro clínico de PC atáxica, observa-se a presença distúrbio da coordenação dos movimentos em razão da dissinergia, manifestando uma marcha com aumento da base de sustentação e tremor intencional, em detrimento da disfunção cerebelar (Rosenbaum et al., 2007). Acredita-se que o quantitativo de casos de PC, representam um total de 1,5 a 2,5 para cada mil nascidos vivos, nos países de primeiro mundo, e de 7 para cada mil nascidos vivos nos países em desenvolvimento. No Brasil, os dados apontam cerca de 30 a 40 mil casos novos por ano (Mancini et al., 2002).

A Fisioterapia nas crianças que apresentam PC, abrange métodos e técnicas capazes de serem utilizadas com abordagem terapêutica nos distúrbios sensório-motores, fazendo uso de terapia por vestes elásticas por meio de protocolos de Terapia Neuromotora Intensiva (TNMI). A utilização dos protocolos de TNMI interligados a Fisioterapia, com períodos consecutivos durante o tratamento, tem como objetivo, promover, restaurar e assegurar a utilidade do corpo como um todo, por meio dos métodos Pediasuit e Therasuit (Neves et al., 2013; Peres et al., 2016). A intervenção fisioterapêutica nestas crianças com PC, pode incluir fortalecimento e treinamento específico, com o propósito de ajudá-las a atingir o seu potencial, em busca de alcançar maiores desenvolvimentos físicos (Bailes et al., 2011).

O benefício do tratamento pode fornecer mudanças necessárias, no entanto as evidências são baseadas sempre em treinamentos intensivos com período de 4 semanas, os efeitos da intensidade, onde não há evidências que quanto maior a intensidade, maiores serão os resultados (Mélo et al., 2017).

O Método Pediasuit é uma abordagem terapêutica de crianças com desordens neurológicas, que apresentam alterações nas funções motoras e cognitivas, almejando manter o alinhamento do corpo, recuperação funcional com peças que são ligadas a cabos de borracha. Utilizando durante as sessões de terapia, uma vestimenta por até quatro horas em cinco dias da semana, na faixa de um mês em que, a maior parte dos protocolos são estabelecido da seguinte maneira: aquecimento, alongamento, vestimenta. A veste é trabalhada contra resistências, aumento de propriocepção, como objetivo de reduzir as sinergias musculares e patológicas (Azevedo, 2014).

Os equipamentos utilizados no decorrer das sessões, quando associados a outros métodos de reabilitação fisioterapêutica, podem contribuir positivamente na evolução do quadro clínico das crianças com PC. Almejando minimizar os reflexos patológicos, utiliza-se em suas estruturas básicas o suit (traje) e o spider, que representa uma gaiola terapêutica (Almeida, et al., 2020).

O Therasuit é constituído por: short, colete, joelheira e uma conexão que se encontra intercomunicada por um sistema de cordas elásticas, com o propósito de promover estabilização, facilitação e dar suporte aos grupos musculares, esse mecanismo produz estímulos sensoriais ao sistema nervoso central, pelos receptores sensoriais presentes em várias estruturas, para discriminar a posição e o movimento articular, inclusive a direção, amplitude, assim como a tensão realizada sobre os tendões (Martimbianco et al.,2008; Ccates, 2015).

O método Therasuit, reflete na melhora da propriocepção, por aferência no sistema nervoso central pelos diversos tipos de receptores sensoriais presentes nas estruturas, inclusive o sentido, amplitude e velocidade (Lemos, et al., 2015). Apesar de existir protocolo padronizado, os treinamentos geralmente não são determinados, podendo haver adaptações, dependendo do paciente e terapeuta (Rémon, et al., 2021).

Com o objetivo de investigar e descrever os efeitos terapêuticos produzidos pelos métodos Pediasuit e Therasuit, em crianças com PC. Foi realizado esta revisão sistemática, almejando identificar as evidências científicas sobre os possíveis ganhos associados ao controle motor, coordenação motora, equilíbrio, força muscular e qualidade de vida. 


\section{Metodologia}

Este estudo, possui uma abordagem quantitativa, utilizando como método científico a revisão sistemática. Este tipo de revisão, apresenta uma investigação científica, destinada a avaliar criticamente e conduzir, uma síntese dos resultados de múltiplos estudos primários. Ela também objetiva responder a uma pergunta claramente formulada, utilizando métodos sistemáticos e explícitos para identificar, selecionar e avaliar as pesquisas relevantes, coletar e analisar dados de estudos incluídos na revisão. Lembrando que os métodos estatísticos podem ou não ser usados para analisar e sumarizar os resultados dos estudos incluídos (Cordeiro, et al., 2008).

Na composição do presente estudo, foi realizado a coleta nas bases de dados: BVS (Biblioteca Virtual em Saúde), PubMed, SCIELO (Scientific Eletronic Library Online) e Periódicos (CAPES/MEC), devido apresentarem publicações nacionais e internacionais destinadas a área da saúde, com um período de busca de agosto a outubro de 2021. As palavraschaves utilizadas foram: paralisia cerebral, vestes elásticas, terapia neuromotora intensiva, pediasuit e therasuit. Utilizando estudos publicados nos idiomas: português, inglês e espanhol; realizando-se, a combinação das palavras-chave com as respectivas línguas, sendo eles: "paralisia cerebral, cerebral palsy e parálisis cerebral”, vestes elásticas, elastic garments e prendas elásticas", "terapia neuromotora intensiva, intensive neuromotor Therapy e terapia neuromotora intensiva”, "pediasuit, pediatric suit e traje pediátrico", e "therasuit, therasuit e therasuit".

Para o processo de construção da referida pesquisa, utilizou-se como critérios de inclusão: resumos publicados em anais de congresso, artigos publicados em periódicos ou online, dissertações e teses nas línguas portuguesa, inglesa e espanhola, desde que apresentar-se uma das palavras chaves em seu título ou que abordasse elementos importantes sobre o tema proposto. Foram descartados, os materiais duplicados e aqueles que não descrevem a paralisia cerebral ou a terapia por vestes elásticas.

Inicialmente, foram identificados, cerca de 21.116 artigos, dos quais, foram agrupados, realizado o cruzamento das palavras-chave, através do operador booleano "AND”, de acordo com cada palavra-chave. Após serem submetidos aos critérios de inclusão e exclusão, restaram cerca de 28 artigos. A partir deste momento, inicia-se a etapa destinada a leitura na íntegra de cada artigo científico, com o objetivo de selecionar as evidências científicas sobre a fisiopatologia da paralisia cerebral e a terapia por vestes elásticas. No fluxograma abaixo, se encontra a sequência das etapas metodológicas.

Fluxograma 1 - Fluxograma dos artigos identificados.

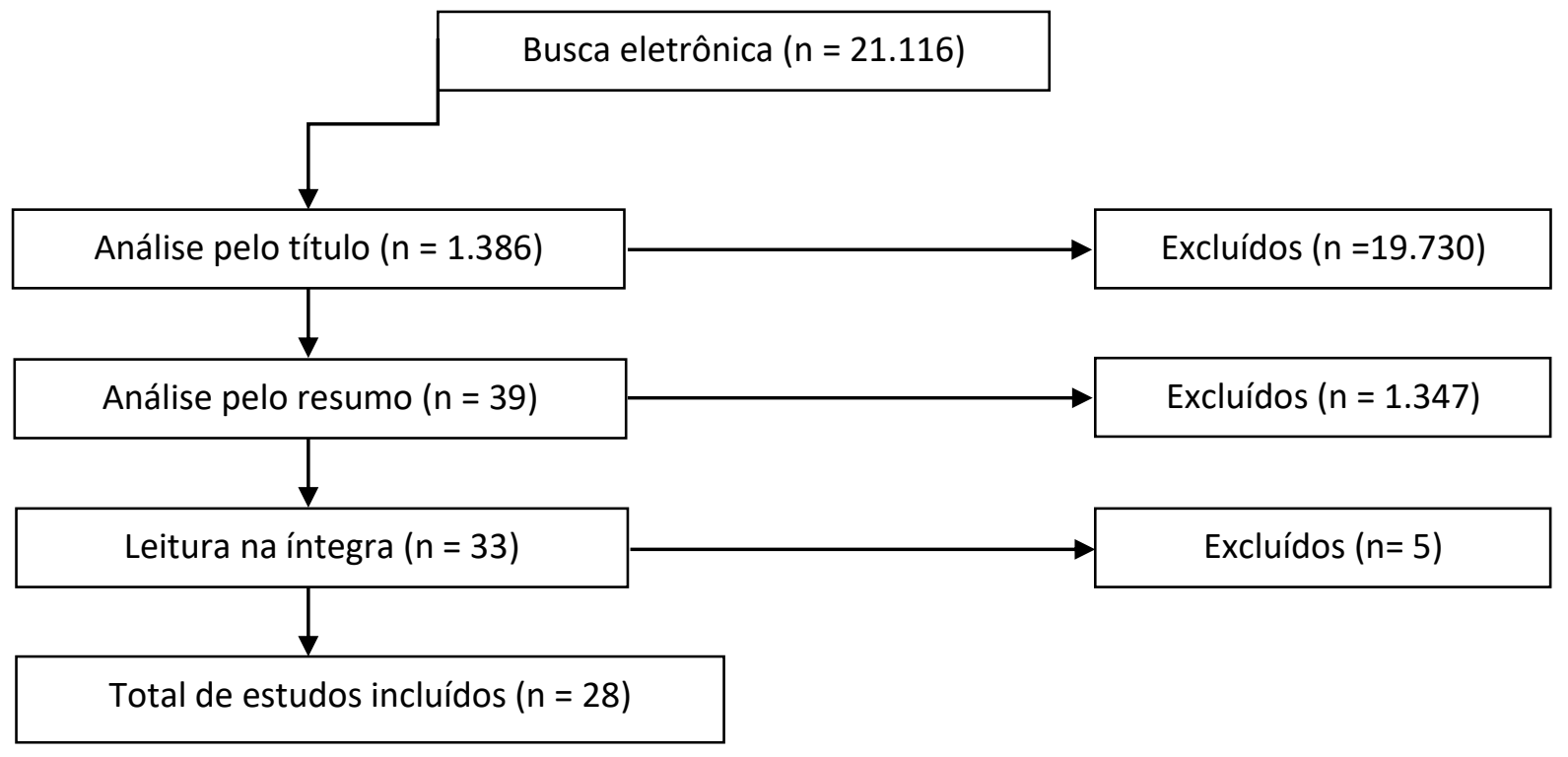

Fonte: Elaboração dos autores (2021). 


\section{Resultados}

Utilizando as palavras-chaves: paralisia cerebral, vestes elásticas, pediasuit, therasuit e terapia neuromotora intensiva; em cada base de dados, foram identificados cerca de 21.116 artigos, assim descrito na Tabela 1.

Quadro 1 - Acervo em cada base de dados científicos.

\begin{tabular}{|l|c|c|c|c|}
\hline Descritores & SciELO & BVS & PubMed & $\begin{array}{c}\text { Periódicos } \\
\text { Capes }\end{array}$ \\
\hline Paralisia Cerebral & 326 & 18.887 & 22 & 1.690 \\
\hline Vestes elásticas & 0 & 2 & 9 & 43 \\
\hline Terapia Neuromotora Intensiva & 1 & 3 & 1 & 61 \\
\hline Pediasuit & 2 & 2 & 3 & 26 \\
\hline Therasuit & 2 & 11 & 5 & 32 \\
\hline
\end{tabular}

Fonte: Elaboração dos autores (2021).

Sabendo que o quantitativo geral dos materiais presentes na literatura, foram cerca de 21.116, destes, foram selecionados cerca de 28 artigos, dos quais, melhor descreveram a fisiopatologia da PC e as técnicas de terapia por vestes elásticas, sendo elas Therasuit e Pediasuit. No quadro abaixo, se encontram exposto, a descrição de cada artigo utilizado abordando: o título, nome do autor e ano de publicação, descritores ou palavras-chave, objetivos, método utilizado e um resumo dos resultados de cada estudo.

Quadro 2 - Síntese dos resultados sobre a utilização da TNMI em crianças com PC.

\begin{tabular}{|c|c|c|c|c|c|}
\hline Título & $\begin{array}{l}\text { Autores e ano } \\
\text { de publicação }\end{array}$ & Palavras-chave & Objetivo da pesquisa & Método & Resultado \\
\hline $\begin{array}{l}\text { Description of the } \\
\text { Pediasuit } \\
\text { Protocol. }\end{array}$ & $\begin{array}{l}\text { SCHEEREN, } \\
\text { Eduardo M. et } \\
\text { al., } 2012 .\end{array}$ & $\begin{array}{l}\text { Pediasuit, Terapia } \\
\text { Intensiva, } \\
\text { Paralisia Cerebral. }\end{array}$ & $\begin{array}{l}\text { Foi descrever o Método } \\
\text { Pediasuit, em uma clínica } \\
\text { com fisioterapeutas treinados } \\
\text { pela equipe Pediasuit } \\
\text { Protocolo. }\end{array}$ & $\begin{array}{l}\text { Estudo de } \\
\text { caso }\end{array}$ & $\begin{array}{l}\text { Percebeu-se que o Método Pediasuit juntamente } \\
\text { com a vestimenta promove resultados positivos } \\
\text { ao longo da fisioterapia tradicional. }\end{array}$ \\
\hline $\begin{array}{c}\text { O } \\
\text { desenvolvimento } \\
\text { da roupa } \\
\text { Biocinética. }\end{array}$ & $\begin{array}{l}\text { PRAZERES, } \\
\text { Larissa S. et al., } \\
2013 .\end{array}$ & $\begin{array}{l}\text { Paralisia Cerebral, } \\
\text { Reabilitação, } \\
\text { Equipamentos de } \\
\text { Autoajuda, } \\
\text { Tecnologia } \\
\text { Assistida. } \\
\end{array}$ & $\begin{array}{lr}\text { A reabilitação } & \text { de crianças } \\
\text { com } & \text { deficiências } \\
\text { neuromotoras. } & \end{array}$ & $\begin{array}{l}\text { Estudo de } \\
\text { caso. }\end{array}$ & $\begin{array}{l}\text { Percebeu-se que as vestimentas possibilitam } \\
\text { maior suporte corporal, favorecimento da } \\
\text { estabilidade, inibição de padrões. }\end{array}$ \\
\hline $\begin{array}{l}\text { Efeitos do } \\
\text { Protocolo } \\
\text { Pediasuit no } \\
\text { tratamento de } \\
\text { crianças com } \\
\text { Paralisia } \\
\text { Cerebral. }\end{array}$ & $\begin{array}{l}\text { SILVA, } \\
\text { Caroline S. et } \\
\text { al., } 2017 .\end{array}$ & $\begin{array}{c}\text { Pedia Suit, } \\
\text { Paralisia Cerebral, } \\
\text { Desenvolvimento } \\
\text { Motor. }\end{array}$ & $\begin{array}{llr}\text { Apresentar } & \text { os } & \text { efeitos da } \\
\text { aplicação } & \text { do } & \text { Protocolo } \\
\text { Pediasuit no } & \text { processo de } \\
\text { reabilitação } & \text { de } & \text { crianças } \\
\text { portadoras } & \text { de } & \text { paralisia } \\
\text { cerebral. } & & \end{array}$ & $\begin{array}{l}\text { Revisão de } \\
\text { literatura. }\end{array}$ & $\begin{array}{l}\text { O método Pediasuit, apresenta-se como um bom } \\
\text { método de reabilitação, onde há maior ganho } \\
\text { funcional, proporcionando melhorias nos } \\
\text { aspectos da função motora, desenvolvimento } \\
\text { motor e postura, principalmente quando } \\
\text { associado a terapia intensiva e gaiola funcional. }\end{array}$ \\
\hline $\begin{array}{l}\text { Método Pediasuit } \\
\text { melhora a função } \\
\text { motora grossa de } \\
\text { criança com } \\
\text { paralisia Cerebral } \\
\quad \text { atáxica. } \\
\end{array}$ & $\begin{array}{l}\text { PIOVEZANI, } \\
\text { Joice C. et al., } \\
\quad 2017 .\end{array}$ & $\begin{array}{l}\text { Paralisia Cerebral, } \\
\text { Terapia Intensiva, } \\
\text { Fisioterapia }\end{array}$ & $\begin{array}{l}\text { Verificar a influência do } \\
\text { Pediasuit na função motora e } \\
\text { na percepção do cuidador de } \\
\text { uma criança com paralisia } \\
\text { cerebral. }\end{array}$ & $\begin{array}{l}\text { Estudo de } \\
\text { caso. }\end{array}$ & $\begin{array}{l}\text { Foi realizado através da escala de GMFM e do } \\
\text { Pediasuit a avaliação e notou-se que houve um } \\
\text { aumento significativo na escala onde, foi mais } \\
\text { evidente a criança em pé, a intervenção resultou } \\
\text { na melhora significativa na função motora grossa } \\
\text { da participante do estudo. }\end{array}$ \\
\hline $\begin{array}{c}\text { Avaliação da } \\
\text { psicomotricidade } \\
\text { em crianças com } \\
\text { encefalopatia } \\
\text { crônica não } \\
\text { progressiva da } \\
\text { infância com Suit } \\
\text { terapia } \\
\text { (Pediasuit). }\end{array}$ & $\begin{array}{l}\text { XAVIER, Ellen } \\
\text { L. et al., } 2018 .\end{array}$ & $\begin{array}{c}\text { ECNPI, } \\
\text { Psicomotricidade, } \\
\text { Suit Terapia. }\end{array}$ & $\begin{array}{l}\text { Avaliar a Psicomotricidade } \\
\text { em crianças com ECNPI com } \\
\text { a utilização da Suit terapia. }\end{array}$ & $\begin{array}{l}\text { Estudo de } \\
\text { caso. }\end{array}$ & $\begin{array}{l}\text { Avaliação da Psicomotricidade em crianças com } \\
\text { ECNPI com a utilização do Pediasuit, obtiveram } \\
\text { perfis normais, e a Pediasuit contribuiu } \\
\text { positivamente para a psicomotricidade em } \\
\text { crianças com ECNPI. }\end{array}$ \\
\hline $\begin{array}{c}\text { O Método } \\
\text { Pediasuit no } \\
\text { desenvolvimento } \\
\text { Neuropsicomotor } \\
\text { em crianças com }\end{array}$ & $\begin{array}{l}\text { SAMPAIO, } \\
\text { Iasmin F. et al., } \\
2018 .\end{array}$ & $\begin{array}{l}\text { Paralisia Cerebral, } \\
\text { Pediasuit, gaiolas } \\
\text { funcionais. }\end{array}$ & $\begin{array}{l}\text { Foi analisar o Método } \\
\text { Pediasuit e a gaiola funcional } \\
\text { em crianças com Paralisia } \\
\text { Cerebral. }\end{array}$ & $\begin{array}{l}\text { Revisão de } \\
\text { Literatura. }\end{array}$ & $\begin{array}{l}\text { A análise do método Pediasuit e gaiola funcional } \\
\text { em crianças com PC para melhorar } \\
\text { condicionamento físico e motor, aponta como } \\
\text { resultado, o método Pediasuit, contribui } \\
\text { positivamente para melhora da funcionalidade de }\end{array}$ \\
\hline
\end{tabular}




\begin{tabular}{|c|c|c|c|c|c|}
\hline Paralisia Cerebral & & & & & crianças com PC. \\
\hline $\begin{array}{l}\text { Efecto del traje } \\
\text { terapêutico em La } \\
\text { funcion motora } \\
\text { gruessa de ninõs } \\
\text { com parálisis } \\
\text { cerebral. }\end{array}$ & $\begin{array}{l}\text { ANDRADE, } \\
\text { Claudia P. C } \text { et } \\
\text { al., } 2018 .\end{array}$ & $\begin{array}{l}\text { Paralisia Cerebral, } \\
\text { traje terapêutico, } \\
\text { Therasuit, Função } \\
\text { motora Grossa. }\end{array}$ & $\begin{array}{l}\text { Tem o objetivo de descrever } \\
\text { as mudanças na função } \\
\text { motora grossa em uma } \\
\text { população pediátrica com } \\
\text { diferentes tipos de paralisia } \\
\text { cerebral tratada com traje } \\
\text { terapêutico. }\end{array}$ & $\begin{array}{l}\text { Revisão de } \\
\text { literatura. }\end{array}$ & $\begin{array}{l}\text { Foi realizado através da escala de GMFM e do } \\
\text { método Pediasuit, foi visto um enfoque } \\
\text { terapêutico efetivo para habilidades motoras } \\
\text { grossas, em crianças com grau de } \\
\text { comprometimento severo e com espasticidades. }\end{array}$ \\
\hline $\begin{array}{l}\text { Método Pediasuit } \\
\text { no tratamento da } \\
\text { paralisia cerebral. }\end{array}$ & $\begin{array}{c}\text { MULLER, } \\
\text { Alessandra B et } \\
\text { al., } 2018 .\end{array}$ & $\begin{array}{l}\text { Paralisia Cerebral, } \\
\text { Therasuit, } \\
\text { Pediasuit, Vestes } \\
\text { terapêuticas, } \\
\text { Terapia } \\
\text { Neuromotora. }\end{array}$ & $\begin{array}{l}\text { Verificar os efeitos do } \\
\text { Método } \\
\text { observando a função motora } \\
\text { grossa e o desempenho } \\
\text { funcional de duas crianças } \\
\text { com PC. }\end{array}$ & $\begin{array}{l}\text { Estudo de } \\
\text { caso. }\end{array}$ & $\begin{array}{l}\text { Ao verificar os efeitos do Pediasuit na função } \\
\text { motora e desempenho funcional de dois meninos } \\
\text { com diagnostico de PC do tipo diparesia e } \\
\text { tetraparesia espástica. Durante } 4 \text { semanas de } \\
\text { tratamento intensivo, com duração de } 3 \mathrm{~h} \text { por dia } \\
\text { favorecendo a função motora grossa e } \\
\text { desempenho funcional, foram constatadas } \\
\text { mudanças expressivas no desempenho motor dos } \\
\text { participantes, entretanto, ambos permaneceram } \\
\text { no mesmo nível de classificação funcional após o } \\
\text { tratamento dos sujeitos avaliados, então não é } \\
\text { possível afirmar uma melhora significativa, pois } \\
\text { seria necessário o acompanhamento por um } \\
\text { período prolongado. }\end{array}$ \\
\hline $\begin{array}{l}\text { Análise dos } \\
\text { efeitos do Método } \\
\text { Therasuit na } \\
\text { função motora de } \\
\text { uma criança com } \\
\text { paralisia cerebral. }\end{array}$ & $\begin{array}{l}\text { OLIVEIRA, } \\
\text { Léia C. et al., } \\
2019 .\end{array}$ & $\begin{array}{l}\text { Paralisia Cerebral, } \\
\text { Desenvolvimento } \\
\text { Infantil, } \\
\text { Reabilitação } \\
\text { neurológica, } \\
\text { Fisioterapia. }\end{array}$ & $\begin{array}{l}\text { Foi analisar os efeitos do } \\
\text { Método Therasuit na função } \\
\text { motora de uma criança com } \\
\text { PC. }\end{array}$ & $\begin{array}{l}\text { Estudo de } \\
\text { caso. }\end{array}$ & $\begin{array}{l}\text { Através da análise dos efeitos do método } \\
\text { Therasuit na função motora de uma criança com } \\
\text { Paralisia pelo protocolo GMFM, com a aplicação } \\
\text { do Método Therasuit, houve aprendizado motor, } \\
\text { identificado pelos scores totais de aprendizado, } \\
\text { mostrando efeitos positivos. }\end{array}$ \\
\hline $\begin{array}{l}\text { Therasuit e } \\
\text { Pediasuit em } \\
\text { crianças com } \\
\text { Paralisia } \\
\text { Cerebral. }\end{array}$ & $\begin{array}{l}\text { ROSA, Kelly R. } \\
\text { R et al., } 2019 .\end{array}$ & $\begin{array}{l}\text { Paralisia cerebral, } \\
\text { therasuit, } \\
\text { pediasuit, vestes } \\
\text { terapêuticas, } \\
\text { terapia } \\
\text { neuromotora } \\
\text { intensiva. } \\
\end{array}$ & $\begin{array}{l}\text { Teve o objetivo de realizar } \\
\text { um levantamento } \\
\text { bibliográfico sobre o trabalho } \\
\text { da TNMI utilizada nos } \\
\text { protocolos Therasuit e } \\
\text { Pediasuit em crianças com } \\
\text { PC. }\end{array}$ & $\begin{array}{l}\text { Revisão de } \\
\text { caráter } \\
\text { integrativo. }\end{array}$ & $\begin{array}{l}\text { Ressaltou a importância da Fisioterapia associada } \\
\text { ao método TNMI, foca em trazer autonomia ao } \\
\text { paciente com PC, associado ao método Pediasuit } \\
\text { e Therasuit, com princípios de resistência, treino } \\
\text { intensivo e habilidades motoras. }\end{array}$ \\
\hline
\end{tabular}

Fonte: Elaboração dos autores (2021).

\section{Discussão}

Na revisão de literatura de Schreen et al., (2012), há relatos de que a PC é composta por um somatório de desordens dos movimentos e posturas, causando limitações que são atribuídas a distúrbios não progressivos que ocorreram no desenvolvimento fetal ou infantil. Os casos estão acompanhados por distúrbios de sensação, percepção, cognição, comunicação e comportamento, incluindo problemas musculoesqueléticos.

Com relação a forma de tratamento Scheeren et al., (2012), descreve que o Método Pediasuit consiste em uma fisioterapia intensiva que é realizada, por até quatro horas de terapia por dia, cinco vezes na semana dividida em quatro etapas: aquecimento, alongamento que ocorre na "Jaula de macaco" e "Jaula de Aranha", onde o traje apresenta a finalidade de ganhar habilidades e fortalecer grupos de músculos responsáveis pelo movimento.

Sampaio et al., (2018), ressalta em seu estudo que as gaiolas podem ser usadas de duas formas, sendo estas: a gaiola de macaco que é composta por roldanas e pesos (utilizada para fortalecer músculos específicos) e gaiola de aranha sendo composta por cinto e cordas elásticas (com a finalidade de auxiliar no posicionamento vertical ou praticar as mais diversas atividades).

Segundo Silva (2017) O traje Pediasuit é uma vestimenta ortopédica que consiste em chapéu, colete, calção, joelheiras e calçados que são interconectados por bandas elásticas. O conceito básico do Pediasuit é uma unidade de suporte, que busca alinhar o corpo o mais próximo do normal possível, com a finalidade de restabelecer o alinhamento corporal e descarga de peso, dos quais, são fatores importantes para normalização do tônus muscular, função sensorial e vestibular.

Com Base no Método Pediasuit Piovezani (2017) realizou um estudo de caso que tinha a finalidade de averiguar a influência do Pediasuit na função motora ampla, representa um estudo de uma criança de 6 anos com PC atáxica, o tratamento 
foi individualizado de acordo com a necessidade da criança, utilizando exercícios preparatórios, exercícios com uso da vestimenta "suit", atividades lúcidas de aquecimento, dissociação de cintura pélvica, equilíbrio e fortalecimento. De acordo com a avaliação que antecede o tratamento e após a terapia, os domínios que tiveram diferença foi sentar, engatinhar e ajoelhar, andar, correr e pular, concluindo que o método Pediasuit resultou em uma melhora significativa na função motora grossa da participante.

No estudo descrito por Xavier et al., (2018) que teve o objetivo de avaliar a Psicomotricidade em crianças com ENCPI com a utilização da Suit Terapia, colaboraram com o estudo cinco crianças, a avaliação baseou em tonicidade, equilíbrio estático e dinâmico ,lateralidade, espaço, praxia global e fina, os resultados foram avaliados pela pontuação obtida pela Classificação de Bateria Psicomotora, foi possível identificar que mediante os dados obtidos, que o Pediasuit é capaz de proporcionar evoluções significativas as habilidades psicomotoras.

Muller et al., (2018) utilizou o Método Pediasuit juntamente a escala de GMFM como meio de avaliação de dois meninos com diagnóstico de PC espástico com a utilização da vestimenta específica. O método Pediasuit favoreceu a função motora grossa e o desempenho funcional das crianças submetidas ao tratamento intensivo, foram identificadas transformações significativas na função motora.

Corroborando com os estudos descritos, Oliveira (2019), ao descrever sobre a escala de GMFM na avaliação da função motora de uma criança com PC, por meios de scores, analisando as posturas: deitar, rolar, sentar-se, engatinhar e ajoelhar, ficar de pé e andar, correr e pular; observou a existência de resultados positivos em relação a função motora, com melhora progressiva nos scores sentar-se e em pé do GMFM após a intervenção. Ainda nesse estudo com referência ao Therasuit foi dito que se trata de um método mais recente, ainda existe discussões a respeito da sua eficácia, algumas vertentes explicam a melhoria do padrão motor associando a terapia intensiva com uso de trajes especiais, estimulando assim o sistema nervoso central, principalmente o sistema proprioceptivo.

Por sua vez, Rosa et al., (2019) ressalta que o desenvolvimento motor, fortalecimento muscular, resistência, flexibilidade, coordenação motora e no equilíbrio, baseado nos princípios de resistência, musculatura e o treino intensivo nas habilidades motoras associada aos métodos de TNMI, onde a fisioterapia juntamente com TNMI, há um relevância positiva por meio de métodos Therasuit e Pediasuit como importante recurso terapêutico.

\section{Conclusão}

Diante dos estudos revistos, foi possível verificar que a PC, é determinada como encefalopatia crônica não progressiva, o qual, ocorre devido a uma lesão no encéfalo em desenvolvimento, comprometendo as funções motoras e a postura. Deixando confirmado através dos estudos revistos que o método Pediasuit e Therasuit no tratamento da PC, favorece e estimula a criança no aspecto funcional, com resultados significativos, como melhora no alinhamento postural, respostas motoras, ganho de habilidade, e melhora no tônus muscular, ocasionando uma melhora na qualidade de vida. Vale ressaltar que o tratamento promove alterações de forma diferente, portanto deve ser individualizado. Ambos os métodos de TNMI, por ser tratar de um método novo no Brasil, existe uma carência de pesquisas que possam elucidar sua eficácia, deixando assim lacunas a serem preenchidas em próximas pesquisas científicas com grupo controle, comparando a sua eficácia, com as outras técnicas da fisioterapia.

\section{Referências}

Almeida, K. C. et al. (2020). Efeitos da terapia neuromotora intensiva com traje pediasuit em pacientes com esclerose múltipla: relato de caso. https://revista.uniandrade.br/index.php/revistauniandrade/article/view/1588. 
Andrade, C. P. \& Roldán A. M. (2018). Efecto del traje fisioterapeutico em La función motora gruesa de ninõs com parálisis cerebral. https://www.medigraphic.com/pdfs/revcubped/cup-2018/cup183f.pdf.

Azevedo, S. M. S. (2014). O efeito do método Therasuit na função motora de uma criança com Paralisia Cerebral.2014. Trabalho de Conclusão de Curso (Graduação em Fisioterapia). Universidade Fernando Pessoa, Porto. https://bdigital.ufp.pt/handle/10284/4336.

Bailes. A. F et al (2011). The effects of Suit wear during an intensive therapy program in children with cerebral palsy. https://journals.lww.com/pedpt/Fulltext/2011/23020/The_Effect_of_Suit_Wear_During_an_Intensive.2.aspx.

CCATES. (2015). Centro Colaborador do SUS Avaliação de Tecnologia e Excelência em Saúde. Indicações de equoterapia, TheraSuit e hidroterapia. http://www.ccates.org.br/content/_pdf/PUB_1434396963.pdf.

Cans, C.M et al. (2007) Recommendations from the SCPE collaborative group for defining and classifying cerebral palsy. Developmental Medicine and Child Neurology, 9, 35-38. Supplement 109.

Cordeiro, A. M. \& Gloria. M. O \& Rentería. J. M \& Guimaraes C. A. (2008). Revisão Sistemática: Uma Revisão Narrativa. https://www.scielo.br/j/rcbc/a/CC6NRNtP3dKLgLPwcgmV6Gf/?format=pdf\&lang=pt.

Gómez, M. E; Umbarila. J. A, Sánchez L. B (2016) Efectos de La terapia física intensiva sobre la función motora de um nino con hemiparesia espástica. http://www.scielo.org.co/pdf/rfmun/v64s1/0120-0011-rfmun-64-s1-00157.pdf.

Lemos. L.C (2015) Indicações de Equoterapia, Therasuit e hidroterapia. http://www.ccates.org.br/indicacoes-da-equoterapia-therasuit-e-hidroterapia/.

Mancini, M. C \& Fiuza, P. M \& Rebelo, J. M. \& Magalhães, L. C. \& Coelho, Z. A. C. \& Paixão, M.L. et al. (2002) Comparação do desempenho de atividades funcionais em crianças com desenvolvimento típico e crianças portadoras de paralisia cerebral. Arq Neuro-Psiquiatr. 60(2B): 446-452

Mangilli, E. M. (2017). Efeitos musculares do Protocolo PediaSuit em crianças com paralisia cerebral espástica. Dissertação (Mestrado em Saúde Coletiva). Universidade do Extremo Sul Catarinense, Criciúma. http://repositorio.unesc.net/handle/1/5240.

Martimbianco, A. L. C. \& POLACHINI, L. O. \& CHAMLIAN, T. R. \& MASIERO, D. (2008). Efeitos da propriocepção no processo de reabilitação das fraturas de quadril. Acta Ortop Bras [online], 16(2),112-116. http://www.scielo.br/scielo.php?pid=S141378522008000200010\&script=sci_abstract\&tlng=pt.

Mélo, T. R; Yamaguchi. B. (2017) Intensive neuro motor therapy with suit improves motor Gross function on cerebral palsy: a Brazilian study. https://revistas.rcaap.pt/motricidade/article/view/13669/10481.

Muller, A. B; Budtinger. L. F. (2018). Método Pediasuit no tratamento da paralisia Cerebral: relato de casos. FisiSenectus. Uno chapecó Ano 6, n. 1 - Jan/Jun. 2018 p. 4-12 . https://bell.unochapeco.edu.br/revistas/index.php/fisisenectus/article/view/3738.

Neves, E. B. \& Krueger, E. \& Pol, S. \& Oliveira, M. C. N. \& Szinke, A. F. \& Rosário, M. O. (2013). Benefícios da terapia neuromotora intensiva (TNMI) para o controle do tronco de crianças com paralisia cerebral. Rev Neurociências. 21(4),549-555.

Oliveira. L. C; Antunes G. L; Gomes M. A. (2019) Análise dos efeitos do Método Therasuit na função motora de uma criança com Paralisia Cerebral. Health Sci Inst. 2019;37(2):165. https://repositorio.unip.br/wp-content/uploads/2020/12/11V37_n2_2019_p165a168.pdf.

Peres, E. F. R. \& Erthal, V. \& Melo, T. R. \& Chiarello, C. \& Neves, E. B. (2016). Avaliação com fotometria de membros inferiores em crianças com paralisia cerebral que fazem tratamento com terapia neuromotora intensiva. In: SEMINÁRIO DE PESQUISA/INICIAÇÃO CIENTÍFICA DA UNIANDRADE.

Piovezani, J. C; Maitschuk. M. M. (2017). Método Pediasuit melhora a função motora grossa de criança com paralisia Cerebral. 10.5585/ConsSaude.v16n1.6689. https://periodicos.uninove.br/saude/article/view/6689/3485.

Prazeres, L. S; Oliveira. A. I. A. (2013). O desenvolvimento da roupa Biocinética. Cad. Ter. Ocup. UFSCar, 21(1), 3-9, 2013 http://dx.doi.org/10.4322/cto.2013.002.

Queiroz. L. et al., (2019). Benefícios do Therasuit no tratamento de encefalopatia crônica não progressiva: revisão de literatura. http://conicsemesp.org.br/anais/files/2019/trabalho-1000003715.pdf

Remón. A. L. C; Panufnick. M. A. (2021). Effectiveness of therapeutic suits and intensive suit trainings in individual swith cerebral palsy and other neurological disorders: a scoping review. https://www.termedia.pl/Effectiveness-of-therapeutic-suits-and-intensive-suit-trainings-in-individuals-with-cerebralpalsy-and-other-neurological-disorders-a-scoping-review,128,42193,1,1.html.

Rosa, K. C. R; Moreira. J. P. (2019) Therasuit e Pediasuit em crianças com Paralisia Cerebral. RRS-FESGO| Vol.02 n.3, pp.102-110 (Ago - Dez 2019) (ISSN online: 2596-3457) https://www.researchgate.net/profile/Luiz-Fernando-Martins-De-Souza- 2021.

Rosenbaum, P.; Stewart, D. (2004). The World Health Organization International Classification of Functioning, Disability and Health: a model to guide clinical thinking, practice and research in the field of cerebral palsy. Seminars in Pediatric Neurology, 11(1), 5-10.

Santos, G. F. L. \& Santos, F. F. \& Martins, F. P. A. (2017). Atuação da fisioterapia na estimulação precoce em crianças com paralisia cerebral. DêCiência em Foco, 1(2). http://revistas.uninorteac.com.br/index.php/DeCienciaemFoco0/article/view/76.

Sampaio, I. F; Guimaraes, P. S. A; Pereira R. G. B. (2019). O método Pediasuit no desenvolvimento Neuropsicomotor em crianças com Paralisia Cerebral. https://repositorio.alfaunipac.com.br/publicacoes/2019/95_o_metodo_pediasuit_no_desenvolvimento_neuropsicomotor_em_criancas_com_.pdf.

Scholtes, V. A. B. et al., (2006). Clinical assessment of spasticity in children with cerebral palsy: a critical review of available instruments. Developmental Medicine and Child Neurology, 48, 64-73. 
Research, Society and Development, v. 11, n. 1, e36811125007, 2022

(CC BY 4.0) | ISSN 2525-3409 | DOI: http://dx.doi.org/10.33448/rsd-v11i1.25007

Schreen, E. M. et al., (2012) Description Of The Pediasuit Protocol. Fisioter. Mov., 25(3), 473-480, https://www.scielo.br/j/fm/a/hky3nwSCv9qhd97zxqndgQg/?format=pdf\&lang=en.

Silva, C. S; Lacerda R. A. M. V. (2017) Efeitos do Protocolo Pediasuit no tratamento de crianças com Paralisia Cerebral.Revista Multidisciplinar do Nordeste Mineiro -Faculdade Presidente Antônio Carlos de Teófilo Otoni - https://revistas.unipacto.com.br/storage/publicacoes/ 2017/efeitos_do_protocolo_pedia_suit_no_tratamento_de_criancas_com_paralisi_93.pdf.

Xavier, E. L; Júnior. R. A. D. S. et al. (2018). Avaliação da Psicomotricidade em crianças com encefalopatia crônica não progressiva da infância com uso de

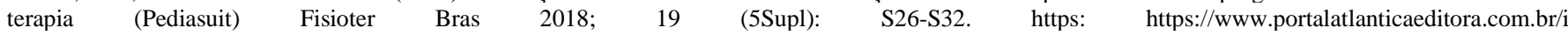
ndex.php/fisioterapiabrasil/article/view/2595/pdf. 